\title{
Survey of isometric scapulothoracic muscles strength in Mazandaran University of Science and Technology athlete's student
}

\author{
Forghani Ozrudi M. ${ }^{1 \mathrm{ABCDE}}$ \\ Young Researchers and Elite Club, Qaemshahr Branch, Islamic Azad University, Qaemshahr, Iran
}

Authors' Contribution: A - Study design; B - Data collection; C - Statistical analysis; D - Manuscript Preparation; E - Funds Collection.

\begin{abstract}
Purpose: $\quad$ Shoulder girdle muscles are important for stabilizing the scapula and orienting the glenoid for upperextremity motion in student athletes. Scapular strength deficits have been linked to shoulder dysfunction.

Material: $\quad$ study the data of scapulothoracic musculature in student athletes using a handheld dynamometer. Cohort study. 66 subjects with varying levels of overhead activity. A handheld dynamometer was used to test the upper, middle, and lower trapezius, rhomboids, and serratus anterior. A one way-factor ANOVA was performed for each of the muscles by activity level analyses. 2-factor ANOVA was performed for each of the muscles by activity level and unilateral ratio by activity-level analyses. Post hoc analysis included multiple pairwise comparisons, using the Dunn-Bonferroni correction method.

Results: $\quad$ Activity level did not significantly affect the unilateral ratios: Elevation: depression was 2.47:1, upward: downward rotation was 1.23:1, and protraction: retraction was 2.35:1. A rank order from strongest to weakest was established through significant comparisons.

Conclusions: The unilateral ratios along with the rank order should be considered when discussing scapula rehabilitation protocols. Assessment of the upper and lower trapezius and serratus anterior muscles and should be part of any shoulder examination.

Keywords: scapulothoracic strength, Muscle activity, scapular dyskinesia, handheld dynamometer.
\end{abstract}

\section{Introduction ${ }^{1}$}

Knowledge of the strength characteristics at a joint can be valuable for a variety of applications. Strength measured from an impaired individual can be compared against healthy strength characteristics to indicate the nature, locality, and severity of musculoskeletal injury. Strength data may be used in engineering applications to design safe and effective products or devices. Finally, strength measurements may be used in research to investigate joint force variability in motor control or to validate computer models representing musculoskeletal biomechanics [1, 2, 3].

Shoulder joint stability is important for the high level of mobility necessary for performance of daily functional tasks and for more challenging athletic activities [4, 5]. Stability of the shoulder relies on static and dynamic restraints such as osseous geometry, integrity of capsuloligamentous supporting structures, the glenoid labrum, as well as muscles involving mechanical and neuromuscular mechanisms. Composed of static and dynamic elements, stability depends on feedback loops from the mechanoreceptors to the central nervous system, termed proprioception, to maintain muscle stiffness and coordination about the joint and thus produce smooth movements. These mechanoreceptors are in the joint, capsules, ligaments, muscles, tendons, and skin [6]. Some authors have argued that alterations in scapular positioning can have an effect on shoulder function [7-9]. Furthermore, scapular positioning is hypothesized to bear a direct relationship to scapular stability and the generation of muscular forces, because coordinated muscle patterns are believed to be requisite for normal glen humeral joint (c) Forghani Ozrudi M., 2019

doi:10.15561/20755279.2019.0302 function and muscle force production $[1,10,11]$. Thus, most authors of texts consider the assessment of scapular position to be one part of a comprehensive approach to evaluation of patients with suspected shoulder dysfunction [12].

The scapula must serve as a stable base for glen humeral function but it also must move through a substantial arc of motion. This motion is required to maintain optimal muscle length-tension relations and glen humeral joint alignment during elevation of the arm [13].

Motion of the scapula on the thorax is essential for normal function of the upper extremity [14]. Specifically, this motion influences glen humeral joint stability, the size of the sub acromial space, and transference of forces from the lower extremities and trunk to the upper extremity [15]. The dynamic constraints of the shoulder girdle include not only the dynamic musculotendinous units of the rotator cuff but also the force couples provided by the scapulothoracicmuscles [13]. The quality of neuromuscular control around the scapula depends on several factors that determine scapular muscle balance [16]. Balanced force production between protractors and retractors is a primary necessity but not the sole condition for muscle balance. In addition, balanced muscle activity among the 3 trapezius parts is necessary for scapular stability. Moreover, balanced timing of muscle recruitment among the scapular muscles is a crucial component of dynamic stability of the scapula throughout arm motion $[13,17,18]$.

Weakness, abnormal positioning, and abnormal timing of the scapular muscles are all contributing factors to scapular dyskinesia. Impairments in scapular motion can lead to problems such as abnormal stresses on the 
anterior capsular structures of the shoulder, increased risk of rotator-cuff compression, and decreased muscle performance [19]. Changes in scapular motion, such as decreased protraction or imbalances between the upper and lower trapezius, have been reported in patients with impingement $[16,18]$. Inadequate scapular stabilization has been shown to contribute to altered biomechanics of the shoulder complex and to increase the risk of musculoskeletal problems such as instability and impingement $[15,18]$.

Amongst the 14 muscles that surround and attach to the scapula, the upper and lower portions of the trapezius and the serratus anterior muscles are believed to be important for scapulothoracic motion. These muscles are typically described as producing upward rotation and retraction of the scapula. Additionally, the upper and lower trapezius and serratus anterior muscles have been proposed to play a role in producing external rotation and posterior tilt of the scapula [14].

Athletes involved in repetitive overhead activities place unique demands on the shoulder girdle [20]. Overhead activities such as throwing, swimming, place the athlete at considerable risk of overuse injuries [17].

The purpose of this study was to study data for strength of the scapulothoracic muscles in healthy individuals, as well as examine the effects of overhead-activity level on these measurements. We hypothesized that those who participate in activities that require increased scapular stabilization, such as overhead athletes, would have higher strength values than those who do not.

\section{Materials and Methods}

Participants.

66 student athletes were recruited from Mazandaran University of Science and Technology sports teams. A cross-section of sports and activities was sampled to examine the differences in subjects with bilateral (swimmers), unilateral (handball players), or no overhead activity (football players). All of the testing took place at team practices or meetings and in the laboratory.

Subjects were asked to fill out a questionnaire regarding their activity level, height, weight, history of previous injury to the upper extremities, and basic demographic information.

\section{Research Design.}

The manual-muscle-testing positions described by Hislop and Montgomery [21]. We measured the isometric strength of the scapulothoracic muscles with the Nicholas Manual Muscle tester (Lafayette Instrument Co, Lafayette IN, model o1160). Muscles rarely work in an isolated manner, so these muscle tests can be considered "biased" toward each muscle. For example, several studies have shown increased middle-trapezius electromyographic activity during the position described for the lower trapezius [22, 23]. However, isolating the middle trapezius' action of scapular retraction is commonly done in clinical practice [21]. Michener et al established construct validity for the lower- and upper-trapezius muscle tests using the positions described respectively [24, 25]. The testing procedure for the serratus anterior targets both functions of the muscle: upward rotation and protraction $[22,23$, 26]. The testing procedure for the rhomboids is illustrated. Smith et al demonstrated that the rhomboid manual muscle test described by Hislop et al was not significantly different than the rhomboid manual muscle test described by Kendall et al when considering the percent maximum voluntary contraction of the rhomboids [25, 27].

For each test, the subject was asked to perform the motion through his or her full range of motion, back off into midrange, and hold the position. A "make" muscle contraction was used rather than a "break" muscle contraction [28]. A make test was used to avoid overpowering the subjects in an effort to measure their force-producing capabilities. Make tests are used almost exclusively with handheld dynamometry [29, 30, 31]. Subjects were asked to build their force gradually to a maximum voluntary effort over a 2 -second period. They maintained a maximum voluntary effort for a 5-second period. The examiner kept the dynamometer in place by matching the force exerted by the subject, and the peak force was recorded. If the subject "broke" against resistance, the data were not recorded, and the muscle test was repeated. Strength measurements were collected for each subject's dominant upper extremity. One trial of each muscle test was used, which has been established in the literature as adequate for measuring muscle strength in healthy subjects $[26,32,33]$. To avoid any possible fatigue factor, all the testing occurred before practices, competitions, or heavy exercise.

The order for testing the muscles was semi randomized to facilitate the speed of testing. Because the end piece of the dynamometer had to be changed, muscle tests requiring the curved end piece were tested together. These muscles included the upper trapezius, serratus anterior, and rhomboids. The middle- and lower-trapezius muscle tests required the rectangular end piece and were therefore tested last.

\section{Reliability Study}

Before actual data collection, we measured the isometric strength of Scapulothoracic muscles in 12 subjects that not included in the study analysis to develop consistent techniques, adopt stable positions for resisting subjects' force, and improve reliability. After sufficient practice, a pilot study was performed to determine the Inter Correlation coefficients (ICC) and Significant Error Means (SEM). Data were analyzed using Statistical Package for the Social Sciences (version 18.0) to calculate interclass coefficients (ICCs) for intrarater and interrater reliability. After ICC calculation, the standard errors of measurement were calculated. See Table 1.

\section{Data Analysis}

Subjects were classified into 1 of the following 3 groups: no overhead activity, unilateral overhead activity, or bilateral overhead activity. No overhead activity was defined as not participating in any athletic activity that required the arm to be elevated above $90^{\circ}$, including, football players, and nonathletic participants. Unilateral overhead activity was defined as participating in any 
athletic activity that required predominately 1 arm to be elevated above $90^{\circ}$, that including handball players. Bilateral overhead activity was defined as participation in athletic activity that required both arms to be elevated above $90^{\circ}$, such as swimmers. Based on the operational definitions used, to be considered for either the unilateral or bilateral athlete's categories, one had to have actively participated in an organized sport for a minimum of 1 year. Descriptive statistics for the demographics for each group are provided in Table 2 .

For each ANOVA, the average force production of each muscle across each activity level was analyzed to determine a rank order for the strength of the 5 muscle groups tested. Then the unilateral strength ratios were determined. The 3 ratios studied were elevation versus depression (upper vs lower trapezius), protraction versus retraction (serratus anterior vs middle trapezius), and upward versus downward rotation (serratus anterior vs rhomboids). For simplicity, the serratus anterior was used to represent upward rotation, instead of the force couple concept using upper trapezius, lower trapezius, and serratus anterior. The middle trapezius was selected because it has the unilateral function of retraction, as opposed to the rhomboids, which have the dual function of retraction and downward rotation. As stated before, muscle weaknesses and imbalances can lead to impingement, so using these unilateral ratios may help establish normal balance of the scapulothoracic muscles [15, 28]. A separate 2-factor ANOVA of ratio by activity level was used to examine the effects of activity level on the strength ratios. Each ratio served as a within-subject factor with 3 levels, and activity level constituted a between-subjects factor with 3 levels.

\section{Procedure}

When significant interactions on main effect were revealed, main interactions, only effects post hoc comparisons were conducted using the Dunn-Bonferroni correction method. Specifically, for significant interactions, only within-group-between muscles and within-musclebetween-groups comparisons were considered. Statistical significance was considered $\mathrm{P}<0.05$.

\section{Results}

The one way-factor ANOVA demonstrated a significant interaction between muscle strength and activity level $(\mathrm{F}=133.58, \mathrm{P}=.001)$ for Upper trapezius, $(\mathrm{F}=115.47$, $\mathrm{P}=.001)$ for Middle trapezius, $(\mathrm{F}=143.07, \mathrm{P}=.001)$ for Lower trapezius, $(\mathrm{F}=391.31, \mathrm{P}=.001)$ for Rhomboids and $(\mathrm{F}=460.38, \mathrm{P}=.001)$ for Serratus anterior muscles. The means and SDs are presented in Table 3.

After a 2-way ANOVA to compare muscle-strength ratios across activity level groups, no significant interaction was observed $(\mathrm{P}=.145)$. A significant main effect for muscle ratio was noted $(\mathrm{P}<.001)$, but the main effect for activity level was not significant $(\mathrm{P}=.601)$. Therefore, it is not necessary to discuss each muscle ratio separately by overhead activity level. Post hoc analysis revealed that the elevation: depression ratio was significantly higher than both of the other ratios. The protraction: retraction ratio was significantly higher than the upward: downward rotation ratio (Table 4 ).

Generally, the overhead athletes (both unilateral and bilateral) had significantly higher muscle strength than the group with no overhead activity. This pattern was true for every muscle except the lower trapezius, which was the weakest across all 3 groups. There were no significant

Table 1. Reliability Analysis for the Pilot Study

\begin{tabular}{lll}
\hline Muscle & ICC & SEM \\
\hline Upper trapezius & 0.86 & 0.43 \\
Middle trapezius & 0.87 & 0.26 \\
Lower trapezius & 0.93 & 0.44 \\
Rhomboids & 0.82 & 0.32 \\
Serrates anterior & 0.89 & 0.37 \\
\hline
\end{tabular}

Table 2. Subjects Classified by Activity Level

\begin{tabular}{llll}
\hline Statistical components & Overhead Activity & & \\
& None & Unilateral & Bilateral \\
\hline Number & 22 & 22 & 22 \\
Mean age (years) & $22.7 \pm 2.31$ & $22.61 \pm 2.53$ & $21.7 \pm 2.3$ \\
Mean height $(\mathrm{cm})$ & $168.31 \pm 6.3$ & $172.73 \pm 6.8$ & $171.59 \pm 5.3$ \\
Mean weight $(\mathrm{kg})$ & $67.4 \pm 8.00$ & $69.81 \pm 6.92$ & $67.82 \pm 7.1$ \\
\hline
\end{tabular}


Table 3. Mean Force Production for Each Muscle Across Overhead-Activity-Level Groups

\begin{tabular}{llll}
\hline Muscle & Overhead activity & Mean(kg) & SD \\
\hline Upper trapezius & Bilateral & 28.85 & 0.869 \\
& Unilateral & 30.46 & 1.14 \\
& None & 25.4 & 1.1 \\
Middle trapezius & Bilateral & 14.15 & 0.46 \\
& Unilateral & 13.87 & 0.50 \\
Nower trapezius & None & 11.72 & 0.73 \\
& Bilateral & 10.25 & 0.48 \\
Unomboids & Unilateral & 12.26 & 0.57 \\
& None & 9.55 & 0.58 \\
Serratus anterior & Bilateral & 15.9 & 0.32 \\
& Unilateral & 17.50 & 0.49 \\
& None & 13.4 & 0.58 \\
\hline
\end{tabular}

Table 4. Ratio Comparison as Grouped by Overhead-Activity Level

\begin{tabular}{lllll}
\hline Components & Overhead activity & Mean $(\mathbf{n})$ & SD & $\mathbf{n}$ \\
\hline \multirow{3}{*}{ Elevation: depression } & None & 2.48 & 1.10 & 22 \\
& Unilateral & 2.28 & 0.97 & 22 \\
& Bilateral & 2.67 & 1.14 & 22 \\
Upward: downward & None & 1.17 & 0.53 & 22 \\
rotation & Unilateral & 1.26 & 0.46 & 22 \\
& Bilateral & 1.28 & 0.38 & 22 \\
Protraction: retraction & None & 2.31 & 0.87 & 22 \\
& Unilateral & 2.37 & 0.44 & 22 \\
& Bilateral & 2.39 & 0.51 & 22 \\
\hline
\end{tabular}

differences between the unilateral and bilateral overheadactivity groups. There were no statistically significant differences found among any of the groups with respect to the lower trapezius (Table 3).

In the no-overhead-activity group the upper trapezius was significantly stronger than any other muscle. Both the middle trapezius and serratus anterior were significantly stronger than the rhomboids and the lower trapezius. There were no significant differences between the middle trapezius and the serratus anterior. In addition, there were no significant differences between the lower trapezius and the rhomboids. In the unilateral overhead-activity group the upper trapezius was significantly stronger than any other muscle. The serratus anterior was significantly stronger than the middle and lower trapezius, as well as the rhomboids. The middle trapezius was significantly stronger than the lower trapezius and rhomboids. There was no significant difference between the lower trapezius and the rhomboids. In the bilateral overhead-activity group the upper trapezius was significantly stronger than any other muscle the middle trapezius was significantly stronger than the lower trapezius and the rhomboids. The serratus anterior was significantly stronger than the middle and lower trapezius, as well as the rhomboids. 
Table 5. Rank Order for Scapulothoracic Muscle Strength Based on Post Hoc Analysis of Overhead-Activity Level

\begin{tabular}{lll}
\hline Overhead Activity & & \\
\hline None & Unilateral & Bilateral \\
Upper trapezius $^{\mathrm{g}}$ & Upper trapezius $^{\mathrm{g}}$ & Upper trapezius $^{\mathrm{g}}$ \\
Serratus anterior or middle Trapezius & Serratus anterior $^{\mathrm{g}}$ & Serratus anteriorg $^{\mathrm{g}}$ \\
Rhomboids or lower trapezius & Middle trapeziusag $^{\mathrm{g}}$ & Middle trapezius $^{\mathrm{g}}$ \\
& Rhomboids or lower trapezius $^{\mathrm{g}}$ & Rhomboids $^{\mathrm{g}}$ \\
& & Lower trapezius \\
\hline
\end{tabular}

Note: ${ }^{g}$ Indicates the strength of this muscle was significantly greater than those below.

The rhomboids were significantly stronger than the lower trapezius, which is a unique finding to the bilateral overhead activity group. However, Table 5 demonstrates a general template for rank ordering the strength of the scapulothoracic muscles based on overhead-activity level.

\section{Discussion}

One way to objectively measure strength in the clinical setting is through the use of a handheld dynamometer, which is more accurate and less subjective than manual muscle testing. The interrater and interrater reliability of handheld dynamometry have been supported in previous studies $[6,27,31]$. The strength of the rater, experience, and tester stability all can alter the reliability of the measurement [6]. However, if these variables are controlled, the handheld dynamometer can be a valuable assessment tool. Adequate assessment of the scapulothoracic musculature is essential to designing better rehabilitation protocols.

Research has demonstrated that some cases of impingement problems have been adequately resolved with rehabilitation protocols involving scapular muscle reeducation and strengthening exercises [19, 34]. By restoring the normal balance of force couples, physical rehabilitation can improve the position and motion of the scapula to decrease impingements and also increase the strength of rotator-cuff muscles [19]. Therefore, outcome assessments in rehabilitation protocols should address these kinematic and muscle-activity alterations to restore normal scapulothoracic and shoulder-complex movements.

This study was the first to compare between scapulothoracic strength using a handheld dynamometer muscle strength and activity level. When comparing muscle strength and activity level, a common pattern was found. The overhead-activity-level groups (unilateral and bilateral) had significantly greater scapulothoracic muscle strength than the group with no overhead activity. This observation can most likely be explained by a training effect. Although direct training of the scapular stabilizers is not commonly seen in training programs, these muscles are a part of the kinetic chain and were therefore active during overhead activity for stabilization. The later aim of this study was to compare between each muscle's strength in the bilateral overhead-activity level.

There were significant differences between each muscle's strength in the bilateral overhead-activity level, which led to establishing a rank order for this group. The rank order is as follows: upper trapezius, serratus anterior, middle trapezius, rhomboids, and lower trapezius. For the remainder of the activity-level groups, an exact rank order could not be determined given the lack of significant differences between muscles, however several trends were observed. These trends were similar to the order established by the bilateral overhead-activity group. Generally, the upper trapezius was the strongest, followed by the serratus anterior and middle trapezius, followed by the rhomboids and lower trapezius.

As previously stated, scapulothoracic muscle weaknesses lead to imbalances that can result in abnormal stabilization and control of the scapula [19]. Identifying weakness that could potentially lead to shoulder dysfunction could potentially lead to a decreased rate of sub acromial impingement. In one study, the rate of shoulder impingement was $55.1 \%$ of 878 patients with shoulder dysfunction [27]. A rank order can be used to identify weak muscles to modify training and conditioning programs with the goal of restoring normal muscle balance. This comparison would be analogous to considering quadriceps-to-hamstring ratios.

However, with respect to the unilateral ratios, no differences were seen across activity level. The 66 normal subjects included in this study demonstrated upper trapezius strength approximately 2.47 times that of the lower trapezius. The ratio between the upward rotators (represented by the serratus anterior) and the downward rotators (rhomboids) was approximately 1.23:1. The ratio between scapular protraction (serratus anterior) and scapular retraction was approximately 2.35:1. We recognize that the scapulothoracic muscles work as synergists to produce scapular motion. Therefore, using 1 muscle to represent each motion may be a limitation and an oversimplification. Nonetheless, we felt it was more appropriate than having muscles with multiple functions. The ratios, which are rounded for each clinical interpretation, were very consistent among this population of healthy subjects. These findings warrant further research into muscle ratios in patients with shoulder 
pathologies, which may identify specific deficits relative to these pathologies. This information can help focus evidence-based rehabilitation programs.

Research has demonstrated that alterations in scapular kinematics are connected to decreased serratus anterior activity, increased upper trapezius muscle activity, and imbalances between the upper and lower trapezius [22]. Research has shown that scapular upward rotation, posterior tilt, and external rotation were decreased in patients with impingement syndrome when compared with healthy subjects. Ludewig and Cook also demonstrated that subjects with symptoms of impingement had significantly more upper trapezius muscle activity and less serratus anterior activity than a control group [2]. Cools et al found that overhead athletes with impingement demonstrated decreased protraction [31]. These studies underline the importance of scapular kinematics and strengthening exercises in shoulder rehabilitation protocols.

There were several limitations of this study. The scapulothoracic muscles are difficult to test with a handheld dynamometer without crossing multiple joints. We decided that it was important to use previously studied and widely accepted manual muscle tests with as few modifications as possible when using the handheld dynamometer. Several studies have demonstrated increased middle-trapezius EMG activity with the position used to test the lower trapezius [22]. The middle trapezius may help eccentrically control upward rotation of the scapula by limiting scapular abduction, which may contribute to this increased activity during the lower-trapezius test [23]. However, isolating the middle trapezius' function as a scapular retractor in $90^{\circ}$ of abduction and neutral rotation (elbow flexed to $90^{\circ}$ ) helps bias the test to measure only the middle trapezius' strength [21]. We used the position presented for several reasons. First, it is the most commonly described position in most manual-muscle-testing texts. Even though the lower trapezius produces high EMG activity in this position, it also is heavily involved with the upward-rotation force couple. Thus, the high EMG activity previously described likely represents the lower trapezius' producing a synergistic activation to prevent scapular rotation. By using the position, we isolated the movement pattern for scapular retraction without the confounding rotations or force-couple co-activations.
In addition, with the particular model of handheld dynamometer used, there were issues when using a rigid end plate instead of a hand. Pain inhibition may have been a factor in our study, because of the shape of handheld-dynamometer force plates (end pieces). Attempts were made to reduce pain inhibition by avoiding placement directly over bony prominences, but maintaining consistency of dynamometer placement limited our ability to avoid this completely. With respect to dynamometer positioning and pain inhibition, any test that did not appear to be the subject's maximal effort was repeated. We feel that any dynamometer with similar pads would yield similar results. However, further research in this area may benefit from using a more comfortable dynamometer.

\section{Conclusion}

The strength of the 5 scapulothoracic muscles was measured in 66 subjects with no history of shoulder dysfunction using a handheld dynamometer. When the subjects were classified by overhead-activity level, a rank order for strength was established in the bilateral overheadactivity group (upper trapezius, serratus anterior, middle trapezius, rhomboids, and lower trapezius).

When the strength of the scapulothoracic muscles as a unilateral ratio was examined, no significant differences occurred across activity levels. The elevation: depression ratio was approximately $2.47: 1$, upward to downward rotation approximately $1.23: 1$, and protraction to retraction approximately $2.35: 1$. The consistency of the ratios across overhead activity levels could provide a practical clinical guideline for establishing exercise progression and meeting discharge criteria. We hope this information can lead to better objective documentation and customized rehabilitation programs based on the objective evidence and normative data.

This information can also be used to help prevent injuries in performance-enhancement programs or as a screening tool. As physical therapy continues to move toward a direct-access setting, a continued commitment to objective documentation, practical clinical guidelines, and evidence-based rehabilitation programs is increasingly important.

\section{Conflict of interest}

The authors declare no conflict of interest. 


\section{References}

1. Garner BA. Designing strength-proportional hydraulic resistance for an elbow flexion-extension exercise machine. Journal of Medical Devices. 2007;1(1):3-13. https://doi.org/10.1115/1.2355684

2. Ludewig PM, Cook TM. Translations of the humerus in persons with shoulder impingement symptoms. Journal of Orthopaedic \& Sports Physical Therapy.2002;32(6):248-59. https://doi.org/10.2519/jospt.2002.32.6.248

3. Mayer F, Horstmann T, Bäurle W, Grau S, Handel M, Dickhuth HH. Diagnostics with isokinetic devices in shoulder measurements--potentials and limits. Isokinetics and exercise science. 2001;9(1):19-25. https://doi.org/10.3233/IES-2001-0059

4. Mcclure PW, Michener LA, Karduna AR. shoulder Function \& 3-dimensional Scapular Kinematics In People With And Without Shoulder Impingement Syndrome. Journal of Orthopaedic \& Sports Physical. 2007;37(7):420-1. https://doi.org/10.1093/ptj/86.8.1075

5. Neumann D. Kinesiology of the Musculoskeletal System: Foundations for Physical Rehabilitation. 3rd Edition. Elsevier eBook on VitalSource, 3rd Edition; 2017.

6. Cools AM, Johansson FR, Cambier DC, Velde AV, Palmans T, Witvrouw EE. Descriptive profile of scapulothoracic position, strength and flexibility variables in adolescent elite tennis players. British journal of sports medicine. 2010;44(9):678-84. http://dx.doi.org/10.1136/bjsm.2009.070128

7. Smith J, Dietrich CT, Kotajarvi BR, Kaufman KR. The effect of scapular protraction on isometric shoulder rotation strength in normal subjects. Journal of shoulder and elbow surgery. 2006;15(3):339-43. https://doi.org/10.1016/j.jse.2005.08.023

8. Smith J, Padgett DJ, Kaufman KR, Harrington SP, An KN, Irby SE. Rhomboid muscle electromyography activity during 3 different manual muscle tests. Archives of physical medicine and rehabilitation. 2004;85(6):987-92. https://doi.org/10.1016/S0003-9993(03)00618-X

9. Yang JL, Jan MH, Hung CJ, Yang PL, Lin JJ. Reduced scapular muscle control and impaired shoulder joint position sense in subjects with chronic shoulder stiffness. Journal of Electromyography and Kinesiology. 2010;20(2):206-11. https://doi.org/10.1016/j.jelekin.2009.04.007

10. Ekstrom RA, Bifulco KM, Lopau CJ, Andersen CF, Gough JR. Comparing the function of the upper and lower parts of the serratus anterior muscle using surface electromyography. Journal of Orthopaedic \& Sports Physical Therapy. 2004;34(5):235-43. https://doi.org/10.2519/jospt.2004.34.5.235

11. Michener LA, Boardman ND, Pidcoe PE. scapular Muscle Tests in Subjects With Shoulder Pain and Functional Loss: Reliability and Construct Validity. Journal of Orthopaedic \& Sports Physical. 2006;36(6):434-5. https://doi.org/10.1093/ptj/85.11.1128

12. Garner BA, Pandy MG. Estimation of musculotendon properties in the human upper limb. Annals of biomedical engineering. 2003;31(2):207-20. https://doi.org/10.1114/1.1540105

13. Pink MM, Tibone JE. The painful shoulder in the swimming athlete. Orthopedic Clinics of North America. 2000;31(2):247-61. https://doi.org/10.1016/S0030-5898(05)70145-0

14. Holzbaur KR, Murray WM, Delp SL. A model of the upper extremity for simulating musculoskeletal surgery and analyzing neuromuscular control. Annals of biomedical engineering. 2005;33(6):829-40. https://doi.org/10.1007/s10439-005-3320-7

15. Hislop H, Montgomery J. Daniels and Worthingham's Muscle Testing: Techniques of Manual Examination. 10th ed. Philadelphia, PA: Elsevier Science; 2018.

16. Peebles L, Norris B. Filling 'gaps' in strength data for design. Applied ergonomics. 2003;34(1):73-88. https://doi.org/10.1016/S0003-6870(02)00073-X

17. Ekstrom RA, Donatelli RA, Soderberg GL. Surface electromyographic analysis of exercises for the trapezius and serratus anterior muscles. Journal of Orthopaedic \& Sports Physical Therapy. 2003;33(5):247-58. https://doi.org/10.2519/jospt.2003.33.5.247

18. Garner BA, Pandy MG. Musculoskeletal model of the upper limb based on the visible human male dataset. Computer methods in biomechanics and biomedical engineering. 2001;4(2):93-126. https://doi.org/10.1080/10255840008908000

19. Thigpen CA, Padua DA, Morgan N, Kreps C, Karas SG. Scapular kinematics during supraspinatus rehabilitation exercise: a comparison of fullcan versus empty-can techniques. The American journal of sports medicine. 2006;34(4):644-52. https://doi.org/10.1177/0363546505281797

20. Bohannon RW. Measuring knee extensor muscle strength. American journal of physical medicine \& rehabilitation. 2001;80(1):13-8. https://www.ncbi.nlm.nih.gov/pubmed/11138949

21. Millar AL, Jasheway PA, Eaton W, Christensen F. A retrospective, descriptive study of shoulder outcomes in outpatient physical therapy. Journal of Orthopaedic \& Sports Physical Therapy. 2006;36(6):403-14. https://doi.org/10.2519/jospt.2006.2101

22. Kibler BW, McMullen J. Scapular dyskinesis and its relation to shoulder pain. JAAOS-Journal of the American Academy of Orthopaedic Surgeons. 2003;11(2):142-51. https://www.ncbi.nlm.nih.gov/pubmed/12670140

23. Ludewig PM, Cook TM. Alterations in shoulder kinematics and associated muscle activity in people with symptoms of shoulder impingement. Physical therapy. 2000;80(3):276-91. https://doi.org/10.1093/ptj/80.3.276

24. Ebaugh DD, Spinelli BA. Scapulothoracic motion and muscle activity during the raising and lowering phases of an overhead reaching task. Journal of Electromyography and Kinesiology. 2010;20(2):199-205. https://doi.org/10.1016/j.jelekin.2009.04.001

25. McClure PW, Michener LA, Sennett BJ, Karduna AR. Direct 3-dimensional measurement of scapular kinematics during dynamic movements in vivo. Journal of shoulder and elbow surgery. 2001;10(3):269-77. https://doi.org/10.1067/mse.2001.112954

26. Moghadam AN, Mohammadi R, Arab AM, Kazamnajad A. The effect of shoulder core exercises on isometric torque of glenohumeral joint movements in healthy young females. Journal of research in medical sciences: the official journal of Isfahan University of Medical Sciences. 2011;16(12):1555. https://www.ncbi.nlm.nih.gov/pmc/ articles/PMC3434896/

27. Turner N, Ferguson K, Mobley BW, Riemann B, Davies G. Establishing normative data on scapulothoracic musculature using handheld dynamometry. Journal of sport rehabilitation. 2009;18(4):502-20. https://doi.org/10.1123/jsr.18.4.502

28. Burkhart SS, Morgan CD, Kibler WB. The disabled 
throwing shoulder: spectrum of pathology Part III: The SICK scapula, scapular dyskinesis, the kinetic chain, and rehabilitation. Arthroscopy. 2003;19(6):641-61. https://doi.org/10.1016/S0749-8063(03)00389-X

29. Burkhart SS, Morgan CD, KiblerWB. The disabled throwing shoulder: spectrum of pathology, part I: pathoanatomy and biomechanics. Arthroscopy.2003;19:404-420. https://doi.org/10.1053/jars.2003.50128

30. ChristouEA, Grossman M, Carlton LG. Modeling variability of force during isometric contractions of the quadriceps femoris. Journal of motor behavior. 2002;34(1):67-81. https://doi.org/10.1080/00222890209601932

31. Cools AM, Witvrouw EE, Mahieu NN, Danneels LA. Isokinetic scapular muscle performance in overhead athletes with and without impingement symptoms. Journal of athletic training. 2005;40(2):104. https://www.ncbi.nlm. nih.gov/pmc/articles/PMC1150223/

32. Ebaugh DD, McClure PW, Karduna AR. Three-dimensional scapulothoracic motion during active and passive arm elevation. Clinical Biomechanics. 2005;20(7):700-9. https://doi.org/10.1016/j.clinbiomech.2005.03.008

33. Ekstrom RA, Soderberg GL, Donatelli RA. Normalization procedures using maximum voluntary isometric contractions for the serratus anterior and trapezius muscles during surface EMG analysis. Journal of Electromyography and Kinesiology. 2005;15(4):418-28. https://doi.org/10.1016/j.jelekin.2004.09.006

34. Wilk KE, Meister K, Andrews JR. Current concepts in the rehabilitation of the overhead throwing athlete. The American journal of sports medicine. 2002;30(1):136-51. https://doi.org/10.1177/03635465020300011201

\section{Information about the author:}

Forghani Ozrudi M.; http://orcid.org/0000-0003-1683-3106; mohammadbagher.forghani@gmail.com; Young Researchers and Elite Club, Qaemshahr Branch, Islamic Azad University; Mazandaran province, Babol City, Education office, Department of physical education, Qaemshahr, Iran.

\section{Cite this article as:}

Forghani Ozrudi M. Survey of isometric scapulothoracic muscles strength in Mazandaran University of Science and Technology athlete's student. Physical education of students, 2019;23(3):112-119.

https://doi.org/10.15561/20755279.2019.0302

This is an Open Access article distributed under the terms of the Creative Commons Attribution License, which permits unrestricted use, distribution, and reproduction in any medium, provided the original work is properly cited http://creativecommons.org/licenses/by/4.0/deed.en

Received: 03.04.2019

Accepted: 07.05.2019; Published: 28.06.2019 